\title{
Neurophysiological, nerve imaging and other techniques to assess chemotherapy-induced peripheral neurotoxicity in the clinical and research settings
}

\author{
Andreas A Argyriou (1) , ${ }^{1}$ Susanna B Park, ${ }^{2}$ Badrul Islam, ${ }^{3}$ Stefano Tamburin (1) , ${ }^{4}$ \\ Roser Velasco, ${ }^{5}$ Paola Alberti, ${ }^{6}$ Jordi Bruna, ${ }^{7}$ Dimitri Psimaras, ${ }^{8}$ Guido Cavaletti, ${ }^{\prime}$ \\ David R Cornblath, ${ }^{10}$ on behalf of the Toxic Neuropathy Consortium (TNC)
}

\begin{abstract}
- Additional material is published online only. To view, please visit the journal online (http://dx.doi.org/10. 1136jnnp-2019-320969).
\end{abstract}

For numbered affiliations see end of article.

Correspondence to Dr Andreas A Argyriou, Neurology, "Saint Andrew's" State General Hospital of Patras, Patras 26335, Greece; andargyriou@yahoo.gr

Received 9 April 2019 Revised 27 May 2019 Accepted 13 June 2019 Published Online First 29 June 2019

Check for updates

(C) Author(s) (or their employer(s)) 2019. No commercial re-use. See rights and permissions. Published by BMJ.

To cite: Argyriou AA, Park SB, Islam B, et al. J Neurol Neurosurg Psychiatry 2019:90:1361-1369.

\begin{abstract}
Chemotherapy-induced peripheral neurotoxicity (CIPN) is a common dose-limiting side effect of several anticancer medications. CIPN may involve multiple areas of the peripheral nervous system from the autonomic and dorsal root ganglia (DRG) to the axon and any peripheral nerve fibre type. Large diameter sensory myelinated $(A \beta)$ fibres are more frequently involved, but motor, small myelinated $(A \delta)$, unmyelinated $(C)$ or autonomic fibres may also be affected. Here, we review the current evidence on techniques for the CIPN assessment in the clinical and experimental settings. Nerve conduction studies (NCS) may be used at the subclinical and early CIPN stage, to assess the extent of large nerve fibre damage and to monitor long-term outcomes, with the sural or dorsal sural nerve as the most informative. The quantitative sensory neurological examination provides valuable data alongside NCS. Quantitative sensory testing and nerve excitability studies add information regarding pathophysiology. Nerve MRI and ultrasound may provide information on enlarged nerve, increased nerve signal intensity and DRG or spinal cord changes. Skin biopsy, corneal confocal microscopy, laserevoked potentials, contact heat-related potentials and microneurography may reveal the extent of damage to small unmyelinated nerve fibres that go undetected by NCS. The information on the role of these latter techniques is preliminary. Hence, the use of multimodal testing is recommended as the optimal CIPN assessment strategy, employing objective NCS and other specialised techniques together with subjective patient-reported outcome measures.
\end{abstract}

\section{INTRODUCTION}

Chemotherapy-induced peripheral neurotoxicity (CIPN) ranks among the most common doselimiting non-haematological toxicities of several anticancer medications. CIPN is a common cause of chemotherapy dose reductions and discontinuations, both of which may lead to less effective cancer treatment. ${ }^{1}$ Currently, there are no agents that either prevent or treat CIPN. ${ }^{2}$

CIPN can affect the peripheral nervous system (PNS) from the cell body to the axon and any of the peripheral nerve fibres types. However, CIPN predominantly produces sensory neuropathy through damage to large diameter sensory myelinated $(\mathrm{A} \beta)$ fibres or dorsal root ganglia (DRG) cells. Nevertheless, other components of the PNS may also be affected in CIPN, including motor fibres, as well as thinly myelinated (A $\delta$ ) and unmyelinated (C) ones, which convey temperature, pain and autonomic afferents. ${ }^{3}$

Nerve conduction studies (NCS) provide objective measures of large $A \beta$ fibre nerve function and are considered necessary to quantify and accurately monitor CIPN by physicians, both neurologists and oncologists, regulatory authorities and pharmaceutical companies dealing with CIPN. ${ }^{3}$ However, in the routine clinical setting, NCS are not easily and widely available.

In addition to NCS, several other methods may evaluate the integrity of the PNS, and many of these techniques have been assessed in the clinical or research CIPN setting. These assessments aim to provide in-depth information concerning the pathophysiology underlying abnormal axonal function. Moreover, in the last decade, nerve imaging techniques have become available and are used in the research setting to offer diagnostic and predictive measures in CIPN. ${ }^{4}$

Providing first a brief description of the neuropathological hallmarks of CIPN, we review techniques currently available to assess CIPN in the clinical and research settings. We then discuss whether these techniques could contribute to the diagnostic sensitivity of conventional techniques, mainly NCS, to assess CIPN and their potential to be used in clinical practice and therapeutic/neuroprotection trials.

\section{SEARCH STRATEGY AND SELECTION CRITERIA}

We performed a systematic search in PubMed and Scopus for relevant original research papers written in English reporting on humans from 1990 up to date with terms: 'chemotherapy-induced peripheral neurotoxicity', 'toxic neuropathy', 'peripheral neuropathy' and 'neurotoxicity syndromes' combined with each chemotherapy agent, such as 'paclitaxel, docetaxel, taxane, cisplatin, oxaliplatin, vincristine, thalidomide, bortezomib and abraxane' and limited to results using terms, 


\section{SENSORY}
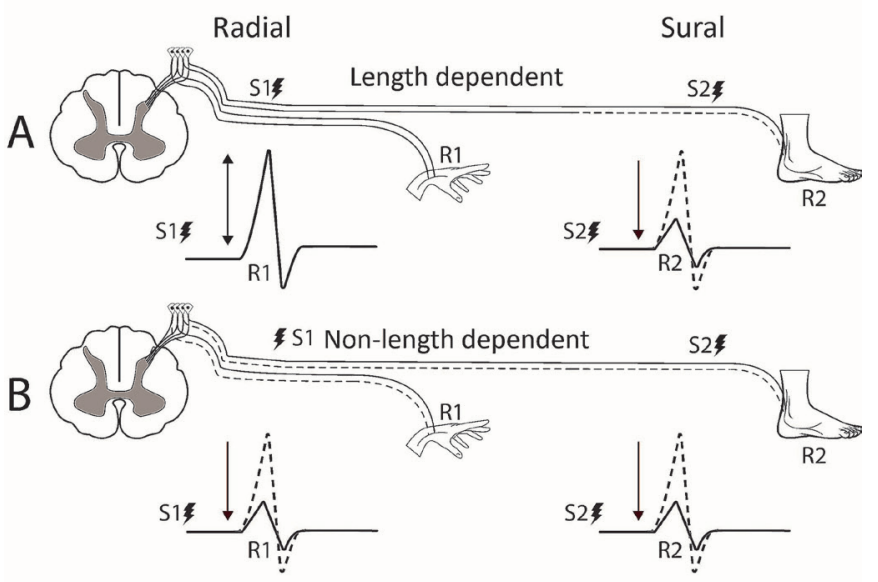

MOTOR
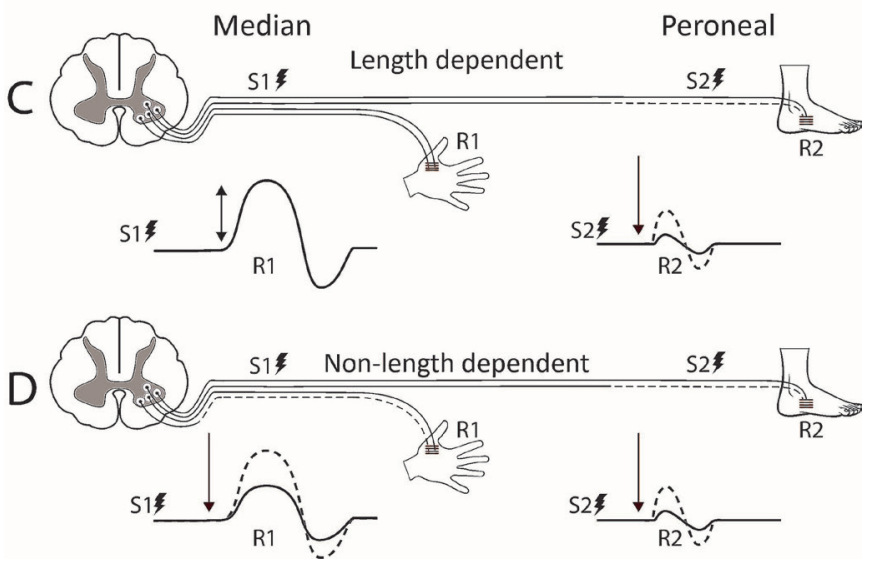

Figure 1 Features of length-dependent versus non-length-dependent neuropathy. (A) Length-dependent sensory neuropathy: Recording of normal radial distal sensory action potential (dSAP) (R1) and low sural dSAP after stimulation at wrist (S1) and ankle (S2). (B) Non-lengthdependent sensory neuropathy: Both low radial and sural dSAP (R1 and R1) recorded after stimulation at wrist (S1) and ankle (S2). (C) Lengthdependent motor neuropathy: Normal median distal compound motor action potential (dCMAP) and low peroneal dCMAP after stimulation at wrist (S1) and ankle (S2). (D) Non-length-dependent motor neuropathy: Low median and peroneal dCMAP after stimulation at wrist (S1) and ankle (S2).

'electrophysiology', 'neurophysiology', 'nerve conduction studies', 'electromyography', 'nerve excitability studies', 'quantitative sensory testing', 'nerve ultrasound', 'sonography', 'ultrasonography', 'nerve MRI', 'skin biopsy', 'microneurography', 'laser-evoked potentials' and 'corneal confocal microscopy'. Single case reports or small case series were excluded for techniques that are commonly used in the clinical setting and with a larger number of studies, while they were included for those that are, to date, less established.

\section{NEUROPATHOLOGICAL HALLMARKS OF CIPN}

In the majority of patients, CIPN is a predominantly sensory neuropathy or neuronopathy. Sensory toxicity predominates over motor toxicity as DRG have fenestrated endothelium cells that allow free passage of molecules between the circulation and the extracellular fluid, and the blood-nerve barrier is much more permeable in DRG than in the spinal cord. ${ }^{5}$
Platinum compounds exert their anticancer effect through binding to the cellular DNA strands and eventually cause cell cycle arrest and apoptotic cell death. ${ }^{6}$ Death of DRG cells results in sensory neuronopathy or ganglionopathy with anterograde neuronal degeneration, which is manifested in NCS as pure sensory non-length-dependent neuropathy affecting both proximal and distal sensory nerve segments (figure 1).

As there is DRG neuronal loss, sensory neuronopathy might be permanent. However, improvement and recovery are seen in some patients following platinum compounds-induced neurotoxicity, thereby suggesting other reversible mechanisms of peripheral nerve damage besides binding to neuronal DNA. Acute and usually reversible sensory neurotoxicity following oxaliplatin can occur in nearly $90 \%$ of cases. In such cases, abnormalities in axonal voltage-gated $\mathrm{Na}+$ channels in the DRG cells through changes in their kinetics or inactivation seem to play a pathogenetic role. ${ }^{78}$

Taxanes, ${ }^{9}$ vinca alkaloids ${ }^{10}$ and bortezomib ${ }^{11}$ produce neurotoxicity mainly by affecting microtubules, thus causing defective axonal transport and a length-dependent 'dying-back' predominantly sensory, axonal polyneuropathy. Mitochondrial dysfunction seems to play a significant role in the pathogenesis of bortezomib-induced neurotoxicity through intracellular calcium homeostasis disruption. ${ }^{11}$ Several other intracellular targets, including decreased $\mathrm{Na}+-\mathrm{K}+$-ATPase-dependent pump function, altered $\mathrm{Na}+$ or $\mathrm{K}+$ conductance and increased tubulin polymerisation, have been suggested. ${ }^{12} 13$

Thalidomide may cause CIPN through microvascular damage in the nerve due to its antiangiogenic property. ${ }^{14}$ Mitochondrial dysfunction and generation of reactive oxygen species have been proposed as mechanisms for most of the neurotoxic agents, which may add to their primary toxic mechanisms. ${ }^{15}$

\section{CONVENTIONAL NCS}

NCS are the gold standard in the diagnosis of CIPN and peripheral neuropathies. ${ }^{16}$ Corresponding to the two main pathogenic mechanisms above, NCS findings in CIPN reveal either a sensory neuronopathy affecting DRGs with a diffuse amplitude decrease or abolishment of sensory action potential (SAP) and normal sensory conduction velocities, or a length-dependent distal, dying-back, axonal degeneration with a reduction of SAP amplitudes in a distal predominant pattern starting in the legs and then involving the arms. Neuronopathy is primarily seen in cisplatin,${ }^{17}$ oxaliplatin ${ }^{18} 19$ and cisplatin plus paclitaxel combination-treated patients, ${ }^{20}$ while a length-dependent axonal sensory neuropathy is the characteristic neurophysiological pattern in taxane, ${ }^{21}$ bortezomib, ${ }^{22}$ thalidomide ${ }^{23}$ and vincristine-exposed patients. ${ }^{24}$ Abnormal SAP have been reported in patients treated with these drugs prior to the symptoms onset, particularly in haematological malignancies, suggesting that they may represent preclinical markers of CIPN. ${ }^{25}$

Primary demyelination with evidence of slowing of motor conduction velocities or F-waves abnormalities is occasionally seen. ${ }^{26}$ Motor involvement is less frequently seen and when present mainly occurs in taxane-treated or vincristine-treated patients with reduced compound muscle action potential amplitudes and electromyographic (EMG) evidence of denervation changes in distal muscles of the lower limbs. ${ }^{24} 27$

NCS of the sural nerve is important when assessing CIPN patients as its early involvement occurs in both neuronopathy and length-dependent neuropathy. Sural NCS provides valuable data in accurately disclosing and following the course of CIPN over time, has predictive value and may differentiate 
Table 1 Quantitative sensory findings in CIPN*

\begin{tabular}{|c|c|c|c|}
\hline Type of chemotherapy & Mechanical sensibility & VDT & CDT/WDT \\
\hline Taxanes & Reduced touch detection ${ }^{542,550}$ & Increased VDT $T^{543-545,548,50}$ & $\begin{array}{l}\text { No change/mildly elevated WDT or } \mathrm{CDT}^{s 42,546-} \\
\text { s48,50 }\end{array}$ \\
\hline Cisplatin & $\begin{array}{l}\text { Reduced touch detection or no change in } \\
\text { some series }{ }^{17}, \mathrm{~s} 112\end{array}$ & Increased VDT ${ }^{17}, \mathrm{~s} 53-\mathrm{s} 55$ & No change/mildly elevated at high dose ${ }^{17}, \mathrm{~s} 112$ \\
\hline Oxaliplatin & $\begin{array}{l}\text { Reduced touch detection in lower limbs or } \\
\text { no change in some series }\end{array}$ & Increased VDT $555,556,5110$ & $\begin{array}{l}\text { Inconsistent findings } \\
\text { (no change, impaired in minority or increased } \\
\text { CDT and WDT) }\end{array}$ \\
\hline Vincristine & Reduced touch detection ${ }^{558,584}$ & Increased VDT ${ }^{\mathrm{s} 111}$ & Increased WDT $T^{558}$ \\
\hline Bortezomib & Reduced touch detection ${ }^{559,560}$ & - & Increased WDT ${ }^{559}$ \\
\hline
\end{tabular}

${ }^{*}$ Cited studies in superscript numbers.

CDT, cold detection threshold;CIPN, chemotherapy-induced peripheral neurotoxicity; VDT, vibration detection threshold; WDT, warm detection threshold.

patients at high risk to develop more severe CIPN. ${ }^{28}$ Similar results were documented in a recent report employing detailed clinical and neurophysiological examination of 200 oxaliplatintreated patients. ${ }^{29}$ The early predictive value of sensory radial and dorsal sural nerve (DSN), to detect patients more prone to severe CIPN, was documented. ${ }^{29}$ Physiologically, the evidence of neuropathic process in the sensory branch of the radial nerve early in the course of chemotherapy supports the neuronopathy pattern of CIPN, because radial nerve sensory function usually remains intact until the late stages of a dying-back neuropathy. ${ }^{30}$

The DSN, a more distal branch of the sural nerve than usually studied in most laboratories, has been suggested to be more sensitive than sural SAP in detecting early dysfunction related to $\mathrm{CIPN}^{31}$ and other neuropathies. ${ }^{32-34}$ DSN NCS was examined in a large international multicenter study of 200 colorectal cancer (CRC) patients monitored before, during and after oxaliplatin chemotherapy. A decreased DSN SAP amplitude, assessed at mid-treatment, was able to predict the neurological outcome at chemotherapy completion. ${ }^{29}$ Longitudinal neurophysiological DSN recordings in 100 CRC patients were used to develop an algorithm, whereby mid-treatment NCS could assign each patient to a 'neurophysiological risk class' for CIPN at the end of treatment with high correlation value. ${ }^{35}$

Although NCS are the most sensitive diagnostic tests for CIPN, they have specific limitations. While they provide valuable data on the integrity of the function of $A \beta$ nerve fibres, they do not provide information about the $\mathrm{A} \delta$ or $\mathrm{C}$ fibres. Nevertheless, NCS are currently considered the gold standard in detecting and monitoring CIPN, with the sural NCS as the most valuable nerve to study. Future NCS studies should include the DSN to increase CIPN detection sensitivity. As there is no validated CIPN biomarker, DSN monitoring might become a valuable tool for risk stratification in clinical practice and research.

\section{AUTONOMIC FUNCTION STUDIES}

The autonomic nervous system (ANS) contains preganglionic myelinated cholinergic fibres arising from the central nervous system to the ganglion and postganglionic fibres arising from the ganglion to the effector organ. Autonomic impairment results in abnormal control of involuntary functions, including heart rate, blood pressure, gastrointestinal motility, urination and perspiration. $^{36}$

Tests are currently available for adrenergic cardiovascular function, parasympathetic cardiovascular innervation and sympathetic sudomotor function. Given that cardiovagal dysfunction is not central to CIPN assessment, the discussion is limited to the assessment of sudomotor function, which can be examined using the quantitative sudomotor axon reflex test (QSART), the sympathetic skin response (SSR) or the Sudoscan device.
QSART requires the application of iontophoresis on the skin, thereby allowing acetylcholine to stimulate eccrine sweat glands. The volume of sweat produced is recorded and compared with normative data. A small study of 13 patients with mixed cancer types and 12 controls $^{37}$ identified significant between-group differences in the number of abnormal QSART sites. However, its results should be cautiously considered because of significant limitations, including the small sample size, the lack of controlling for confounders of ANS function and the heterogeneity of cancer and chemotherapy types.

One study prospectively examined whether SSR could assess the sympathetic sudomotor fibre viability in 23 cisplatin-treated or paclitaxel-treated patients compared with 23 healthy controls and found a significant prolongation of foot and hand SSR latency scores in patients compared with controls. However, no patients showed abnormal SSR in the presence of normal sensory NCS. Hence, the authors concluded that sudomotor function is not preferentially affected than somatic fibres in CIPN. ${ }^{38}$

Sudoscan, a non-invasive method to quantify the electrochemical skin conductance of limbs, has been applied in a single study enrolling 88 patients receiving various types of chemotherapy to assess sudomotor function to detect and follow-up CIPN. This study supported the use of Sudoscan to assess small fibre neuropathy in patients receiving chemotherapy, but results were too preliminary and obtained just from a single setting to be generalised. $^{39}$

To date, there is little evidence to support the use of ANS testing in CIPN, as they yield inferior sensitivity than NCS. Moreover, there are well-known confounders affecting the interpretation of autonomic testing, including age, paraneoplastic involvement and precancer comorbidities, such as diabetes and heart disease. In any case, the autonomic ganglia, similar to the DRG, are less protected by the blood-nerve barrier, so autonomic dysfunction may occur in CIPN. ${ }^{s 40}$ The lower frequency of autonomic symptoms/signs can be attributed to their silent and non-specific nature and the limited availability of autonomic testing in comparison to NCS.

Further studies with adequately powered samples are needed to clarify the mechanism of alterations in sudomotor reactivity. They will also address the clinically relevant question that whether autonomic abnormalities may be found earlier than or always appear after large/somatic nerve fibre damage?

\section{QUANTITATIVE SENSORY TESTING}

Quantitative sensory testing (QST) quantifies subjective sensory function, including thermal and pain thresholds associated with $C$ and $A \delta$ fibres and $A \beta$ fiber-associated touch and vibration thresholds. QST has been utilised in CIPN to determine chemotherapy-specific profiles of sensory dysfunction (table 1), 
Table 2 Characteristics of studies evaluating nerve imaging techniques in CIPN

\begin{tabular}{|c|c|c|c|c|c|c|}
\hline Publication & Study type & Subjects & Cancer & Chemotherapy & Technique & Main findings \\
\hline $\begin{array}{l}\text { Isoardo et al, } \\
2004^{5105}\end{array}$ & CS & 5 & Multiple myeloma & Thalidomide & 1T spinal MRI & $\begin{array}{l}\text { High T2-weighted signal in the posterior } \\
\text { column in parallel with somatosensory } \\
\text { evoked potential changes in } 1 / 5 \text { patients }\end{array}$ \\
\hline $\begin{array}{l}\text { Ravaglia et al, } \\
2008^{s 102}\end{array}$ & CS & 5 & Multiple myeloma & Bortezomib & Spinal MRI & $\begin{array}{l}\text { Lumbar root enhancement in } 2 / 5 \text { patients } \\
\text { who developed severe peripheral } \\
\text { neuropathy with motor involvement }\end{array}$ \\
\hline $\begin{array}{l}\text { Apostolidis et al, } \\
2017^{\text {s103 }}\end{array}$ & CS & 20 & Various types & Oxaliplatin & $\begin{array}{l}\text { 3T MRN } \\
\text { 3T nerve DTI }\end{array}$ & $\begin{array}{l}\text { Significant DRG hypertrophy; minor } \\
\text { morphological qualitative alterations of } \\
\text { peripheral nerves; no quantitative changes } \\
\text { in sciatic CSA and T2-weighted signal; } \\
\text { non-significant diffusivity decrease and no } \\
\text { FA changes }\end{array}$ \\
\hline $\begin{array}{l}\text { Gimber et al, } \\
2019^{\text {s100 }}\end{array}$ & CS & 9 & Various types & Not specified & 3T nerve DTI & $\begin{array}{l}\text { FA and } A D C \text { values did not differ in patients } \\
\text { with versus without CIPN; FA and ADC } \\
\text { values at specific sites were correlated } \\
\text { with VPT }\end{array}$ \\
\hline $\begin{array}{l}\text { Boland et al, } \\
2014^{\text {s106 }}\end{array}$ & CS & $\begin{array}{l}12 \text { patients and } \\
12 \text { healthy } \\
\text { controls }\end{array}$ & Multiple myeloma & $\begin{array}{l}\text { Thalidomide, } \\
\text { bortezomib or } \\
\text { vincristine }\end{array}$ & 3T BOLD brain fMRI & $\begin{array}{l}\text { Greater activation in the precuneus, } \\
\text { reduced activation in the right superior } \\
\text { frontal gyrus to pain stimulation in } \\
\text { patients versus controls; significant } \\
\text { positive correlation between the total } \\
\text { neuropathy score and activation in the } \\
\text { frontal operculum during pain stimulation } \\
\text { in patients }\end{array}$ \\
\hline Briani et al, $2013^{598}$ & CS & 15 & Colorectal & Oxaliplatin & Nerve HRUS & $\begin{array}{l}\text { Sensory axonal neuropathy in } 13 / 15 \\
\text { patients; nerve CSA was increased at } \\
\text { common entrapment sites with no NCS } \\
\text { evidence of entrapment in } 9 / 15 \text { patients }\end{array}$ \\
\hline $\begin{array}{l}\text { Pitarokoili et al, } \\
2019^{999}\end{array}$ & Prospective & 13 & $\begin{array}{l}\text { Pancreatic } \\
\text { (metastatic) }\end{array}$ & FOLFIRINOX & Nerve HRUS & $\begin{array}{l}\text { Length-dependent sensorimotor axonal } \\
\text { neuropathy in } 8 / 13 \text { patients; increased CSA } \\
\text { at upper-limb entrapment sites and tibial } \\
\text { and fibular nerve CSA enlargement; CSA } \\
\text { increased in parallel/prior to clinical signs } \\
\text { and NCS changes; no correlation between } \\
\text { HRUS and NCS abnormalities }\end{array}$ \\
\hline
\end{tabular}

Only studies with more than one patient were reported (ie, no single case reports) in the table.

ADC, apparent diffusion coefficient; BOLD, blood-oxygen-level-dependent; CIPN, chemotherapy-induced peripheral neuropathy; CS, cross-sectional; CSA, cross-sectional area; DRG, dorsal root ganglion; DTI, diffusion tensor imaging; FA, fractional anisotrophy; fMRI, functional MRI; FOLFIRINOX, oxaliplatin, irinotecan and 5-fluorouracil; HRUS, highresolution ultrasound; MRN, magnetic resonance neurography; NCS, nerve conduction study; VPT, vibratory perception threshold.

and in the context of clinical trials, as an outcome measure. In QST studies, CIPN is typically associated with prominent large A $\beta$ fibre loss. A large study of patients with CIPN identified increased vibration thresholds and reduced cold and light touch detection, suggesting some small fibre involvement depending on chemotherapy type. ${ }^{\text {s41 }}$

Paclitaxel-treated patients demonstrated reduced mechanical sensibility, suggesting impairment of A $\beta$ fibres. ${ }^{542}$ Similarly, vibration detection threshold (VDT) was markedly increased in paclitaxel-treated patients, ${ }^{\text {s43 }}$ particularly in distal lower limbs, also indicative of large fibre dysfunction. ${ }^{44}$ VDT has been significantly correlated to patient symptom report of neuropathy. ${ }^{\mathrm{s}}{ }^{45}$

Vibration loss was typically more prominent than changes in thermal perception in paclitaxel-treated patients. ${ }^{.42,546}$ Some reports indicate preserved thermal detection thresholds indicating minimal $\mathrm{C}$ fibre involvement with minor deficits in sharpness detection and paradoxical burning pain indicative of $\mathrm{A} \delta$ fibre impairment. ${ }^{s 42}$ There are also reports of mildly elevated cooling and warming thresholds. ${ }^{s 47}$ Reduced thermal sensation and hot-cold discrimination in lower limbs developed by the final cycle of paclitaxel treatment, ${ }^{s 48}$ suggesting that greater doses may be required to produce thermal deficits.

Similar findings have been identified in docetaxel-treated patients, with dose-dependent vibration $\operatorname{loss}^{\text {s49 }}$ and abnormal mechanical detection threshold, but elevated warm detection threshold (WDT) only in a minority of patients. ${ }^{550}$ Similar findings have also been found in patients treated with both paclitaxel and cisplatin, with prominent reduction in lower limb VDT ${ }^{551}$ and milder deficits in upper limbs. ${ }^{\mathrm{s}}{ }^{2}$

Platinum-treated patients displayed a similar profile, with prominent vibration loss, while thermal detection thresholds were less significantly affected. ${ }^{17}$ In cisplatin-treated patients, the increase in VDT was age-dependent and dose-dependent. ${ }^{553}$ VDT changes persisted long-term in platinum-treated patients, with evidence of recovery in hands but not in feet at 3-4 years post-treatment. ${ }^{\text {s54,s55 }}$

While VDT abnormalities were not evident during early oxaliplatin treatment, increased VDT in the hands and feet during oxaliplatin treatment has been identified as a sensitive marker of neuropathy severity. ${ }^{556}$ Further, both cold and WDTs were increased at 6 months postoxaliplatin completion, but these changes were not linked to the severity of neuropathy. ${ }^{\text {.57 }}$

Vincristine-treated patients also displayed impaired touch thresholds, in addition to deficits in pinprick perception and WDT, indicative of damage to $\mathrm{A} \beta$, A $\delta$ and C fibres. ${ }^{558}$ Similarly, bortezomib-induced reduced touch detection threshold, sharpness detection and increased warmth and heat detection thresholds suggesting involvement of $\mathrm{A} \beta$, A $\delta$ and $\mathrm{C}$ fibres. ${ }^{559}$ 
Table 3 Core characteristics of all available techniques to assess CIPN

\begin{tabular}{|c|c|c|c|c|}
\hline Technique & Structure(s) explored & Main abnormalities & Advantages & Disadvantages \\
\hline $\begin{array}{l}\text { NCS and needle EMG } \\
\text { DSN }\end{array}$ & $\begin{array}{l}\text { A } \beta \text { fibre } \\
\text { DRG }\end{array}$ & $\begin{array}{l}\text { Reduced SAP/CMAP } \\
\text { Reduced MNCV/SNCV } \\
\text { Delayed F-waves } \\
\text { Denervation }\end{array}$ & $\begin{array}{l}\text { Available in all neurological centres } \\
\text { Sural SAP is considered the gold } \\
\text { standard for axonal/DRG damage in } \\
\text { CIPN } \\
\text { DSN SAP more sensitive than sural } \\
\text { SAP }\end{array}$ & $\begin{array}{l}\text { Does not offer information on gain of function } \\
\text { and A-delta and C fibre that may contribute to } \\
\text { neuropathic pain }\end{array}$ \\
\hline Autonomic testing & $A \delta$ and $C$ fibre & $\begin{array}{l}\text { Abnormal QSART } \\
\text { Abnormal SSR }\end{array}$ & $\begin{array}{l}\text { May explore } A \delta \text { and } C \text { fibre that go } \\
\text { undetected with NCS }\end{array}$ & $\begin{array}{l}\text { Available in few centres; less sensitive than } \\
\text { NCS; potential confounders }\end{array}$ \\
\hline LEP and CHEP & $A \delta$ and $C$ fibre & $\begin{array}{l}\text { Reduced LEP and CHEP } \\
\text { amplitude }\end{array}$ & $\begin{array}{l}\text { May explore A } \delta \text { and C fibre that go } \\
\text { undetected with NCS }\end{array}$ & $\begin{array}{l}\text { Available in few centres; time-consuming; C } \\
\text { fibre LEPs are technically difficult; does not } \\
\text { offer information on the site of dysfunction }\end{array}$ \\
\hline $\mathrm{CCM}$ & $\begin{array}{l}\mathrm{A} \delta \text { and } \mathrm{C} \text { fibre in the } \\
\text { cornea }\end{array}$ & $\begin{array}{l}\text { Abnormalities in corneal } \\
\text { nerve fibre }\end{array}$ & $\begin{array}{l}\text { May non-invasively explore } A \delta \text { and } \\
\text { C fibre }\end{array}$ & $\begin{array}{l}\text { Preliminary data on CIPN; no correlation with } \\
\text { clinical and NCS data; correlation with IEFND } \\
\text { not explored }\end{array}$ \\
\hline MRI, MRN and DTI & $\begin{array}{l}\text { DRG } \\
\text { Nerve morphology }\end{array}$ & $\begin{array}{l}\text { Hyperintense or enlarged } \\
\text { nerve } \\
\text { Abnormal FA, diffusivity } \\
\text { and ADC }\end{array}$ & $\begin{array}{l}\text { May offer anatomical information } \\
\text { May explore spinal cord changes }\end{array}$ & $\begin{array}{l}\text { Available in very few centres; time-consuming; } \\
\text { expensive; no information on fibres involved }\end{array}$ \\
\hline HRUS & Nerve morphology & Increased CSA & $\begin{array}{l}\text { May offer anatomical information } \\
\text { Non-invasive and easily applied }\end{array}$ & $\begin{array}{l}\text { Available in few centres; no information on } \\
\text { fibres involved }\end{array}$ \\
\hline
\end{tabular}

On follow-up, 13 months later, deficits persisted but thermal thresholds had partially normalised. ${ }^{560}$ Ixabepilone-treated patients, who developed more severe neuropathy, demonstrated significantly greater increase in VDT from baseline to cycle 2, suggesting that vibration threshold may provide a prognostic tool in this patient group. ${ }^{\text {s61 }}$

While there is robust evidence of QST abnormalities associated with chemotherapy treatment across multiple studies, the utility and value of QST in day-to-day clinical practice still remains to be defined. QST provides insights into sensory function often inaccessible through other techniques; however, it lacks objectivity compared with other assessment methods, such as NCS. Further, QST is dependent on patient cooperation and can be affected by patient-related factors, including cognition and attention. Lastly, QST is a non-specific indicator of pathophysiology with abnormal findings potentially linked to multiple pathologies. Accordingly, while QST provides complementary information regarding impaired sensation, it should be utilised in the context of other clinical findings rather than as a diagnostic tool. ${ }^{\mathrm{s} 62}$

\section{QUANTITATIVE SENSORY NEUROLOGICAL EXAMINATION}

In the absence of QST and NCS devices, other easily applied quantitative sensory neurological examinations (QSNEs) have been implemented in CIPN patients. In a secondary analysis of the chemotherapy-induced peripheral neuropathy outcome measures standardization study (CI-PeriNomS) ${ }^{\mathrm{s} 63}$ data set of 218 patients, several clinical variables were compared with NCS parameters to evaluate their interrelation and correspondence in assessing CIPN. Individual clinical measures included assessment of deep tendon reflexes, muscle strength, vibration sense using the graduated Rydel-Seiffer tuning fork, light touch using standardised $10 \mathrm{~g}$ monofilaments contained within the Neuropen, pinprick sensibility using the disposable Neurotip and strength using the Medical Research Council criteria. Analysis revealed the good correspondence of vibration and light touch abnormalities in lower limbs with sural SAPs. ${ }^{664}$ Abnormalities in vibration sense using a Rydel-Seiffer tuning fork were found predictive of the final neurological outcome in other 62 patients with CIPN. ${ }^{565}$ Hence, traditional bedside examination with these techniques seems to be quite informative in assessing CIPN in settings lacking NCS or more sophisticated tools.

However, the lack of control and standardisation of the stimulus intensity together with its mostly qualitative nature are the main limitations of QSNEs. Hence, future studies might evaluate an expanded set of ranges of stimulus testing as the Rydel-Seiffer tuning fork only tests $64 \mathrm{~Hz}$. In addition, the use of Neuropen, with only one $10 \mathrm{~g}$ filament, clearly reduces the sensitivity and discrimination of this mechanoreceptive test and therefore, the 
use of Von Frey (Semmes-Weinstein) filaments might be preferable in testing painful CIPN cases. ${ }^{\text {s6 }}$

\section{NERVE EXCITABILITY STUDIES}

Nerve excitability studies provide surrogate markers of membrane potential and ion channel function in large myelinated axons, revealing complementary information to conventional NCS on membrane excitability features. Several excitability studies have been undertaken in oxaliplatin-treated patients, examining both acute and chronic neurotoxicity profiles in sensory and motor axons. In addition, insights into axonal excitability in acute oxaliplatin neurotoxicity have been provided by EMG studies. Spontaneous high-frequency motor unit activity is evident on needle EMG, ${ }^{667}$ in addition to repetitive discharges, similar to findings in neuromyotonia and suggesting hyperexcitability. Spontaneous high-frequency action potentials were identified in all patients 2 days postoxaliplatin and were still evident 2 weeks later in $25 \%$ of patients. ${ }^{\text {s67 }}$

Similarly, excitability change is evident in motor axons acutely following oxaliplatin administration using threshold tracking nerve excitability studies. Motor nerves demonstrate prominently increased refractoriness and reduced superexcitability, while sensory axons demonstrate small superexcitability reductions. ${ }^{\text {s68,s69 }}$ Motor and sensory acute excitability changes were linked, so that patients with the greatest changes in sensory axons also demonstrated extensive motor abnormalities. ${ }^{\text {s69 }}$ These acute motor nerve excitability changes suggest alterations in sodium channel function, proposed as a mechanism mediating oxaliplatin acute neurotoxicity in vitro. ${ }^{\text {s70 }}$

Chronic changes in sensory nerve excitability were also evident in oxaliplatin-treated patients, with progressive changes across the treatment. These changes occurred in early treatment, prior to reduction in peak amplitude and were predictive of the severity of chronic neuropathy. In contrast, motor axonal excitability remained unchanged across the treatment. ${ }^{\mathrm{s} 9}$ At follow-up, 2 years later, sensory excitability abnormalities had partially normalised but some deficits remained, ${ }^{\text {s71 }}$ suggesting that they may provide a marker of long-term dysfunction.

Excitability studies in paclitaxel-treated patients demonstrated progressive increase in stimulus threshold and decline in sensory amplitudes without other alterations in sensory axon excitability. ${ }^{547}$ Excitability studies in bortezomib-treated patients demonstrated evidence of axonal depolarisation occurring before sensory axonal loss. ${ }^{\text {s72 }}$ Similar but lesser changes occurred in motor axons, as early as after the first course of administration, suggesting that they may offer early identification of neurotoxicity. ${ }^{\text {s72 }}$

However, excitability studies require special equipment and trained personnel. Therefore, they remain extremely valuable for dissecting pathophysiology but their use in clinical practice is very limited.

\section{MICRONEUROGRAPHY}

Microneurography is an invasive and technically demanding neurophysiological technique. Its main advantage is the direct recording of unmyelinated postganglionic sympathetic or afferent nociceptive fibres by tungsten needles inserted into a peripheral nerve fascicle. Microneurography is mainly applied to disclose abnormal nociceptor outflow underlying pain dysfunctions in PNS. ${ }^{.73}$ Microneurography has demonstrated that a significantly larger proportion of nociceptive $\mathrm{C}$ fibres in the peripheral nerves of patients with high ongoing pain levels are spontaneously active compared with neuropathy patients without continuous pain. This spontaneous activity was suggested to be a major contributor to neuropathic pain. ${ }^{574}$

Microneurography has rarely been utilised in the CIPN research setting. Recently, a patient suffering from severe neuropathic pain treated with lacosamide has been described. ${ }^{\mathrm{s} 75}$ The effect of lacosamide was shown by lowered pain ratings and its peripheral site of action by reduced spontaneous activity of peripheral nociceptors via microneurography. Thus, microneurography might be a helpful tool and objective findings support the assumption that the treatment success is not merely based on placebo effect. However, it should be noted that while interesting, this single patient study needs confirmation in a larger group.

Considering its technical difficulties and the need for extremely skilled physicians, microneurography will remain outside everyday CIPN assessment. However, its use in research areas, such as drug/neuroprotection studies, may be possible.

\section{SKIN BIOPSY}

Skin biopsy allows evaluation of the distal innervation of the skin by the terminal nerve endings of either $\mathrm{A} \delta$ or $\mathrm{C}$ fibres, measuring the intraepidermal nerve fibre (IENF) density. A skin punch biopsy, usually $3 \mathrm{~mm}$ in diameter, can be taken from the proximal thigh $20 \mathrm{~cm}$ below the iliac spine, which in combination with the distal biopsy site $(10 \mathrm{~cm}$ proximal to the lateral malleolus) contributes to differentiation between a neuropathy and a neuronopathy, due to the decreasing density of nerve fibres from the proximal to the distal regions of the body. ${ }^{576,577}$ Loss of IENF is considered a marker of axonal degeneration, and usually, a later event occurring in peripheral nerves, as a consequence of injury in sensory nerve fibres or DRG neurons. ${ }^{\text {s78 }}$

Ten studies totalling less than $150 \mathrm{CIPN}$ patients have incorporated skin biopsy in assessing CIPN (supplementary table). Conflicting results emerge from these small and heterogeneous designed studies such that uniform conclusions regarding the involvement of small fibres with each agent cannot be drawn. ${ }^{\text {s79- }}$ ${ }^{s 88}$ Of note, in two studies, the investigation of the pattern of nerve damage (neuropathy vs neuronopathy) according to the degree of IENF loss in distal and proximal biopsies failed to demonstrate a neuronopathy pattern in oxaliplatin-induced neuropathy. ${ }^{\mathrm{s} 81, \mathrm{~s} 85}$ Most of the studies failed to find a direct association between neuropathic pain and skin biopsy findings. ${ }^{\text {s86-s88 }}$

Nevertheless, skin biopsy is considered the gold standard for diagnosing small fibre neuropathies. However, the contradictory results and methodological issues support further investigation to better define the role of this minimally invasive technique for the assessment of CIPN in clinical practice.

\section{LASER-EVOKED POTENTIALS AND HEAT-RELATED EVOKED POTENTIALS}

Pain-related evoked potentials can be generated by either radiant heat (laser-evoked potentials (LEPs)) or contact heat. LEPs selectively excite free nerve endings in the superficial skin layers, activate $\mathrm{A} \delta$ and $\mathrm{C}$ nociceptors and evoke scalp potentials related to small myelinated fibres. LEPs can document sparing of small myelinated fibres in patients with exclusive damage of the large myelinated fibres, as well as disclose selective dysfunction of nociceptive pathways and their results can be well correlated with the evaluation of IENF features in skin biopsy. ${ }^{\text {s }}{ }^{8}$

The experience of LEPs in CIPN is quite limited as they have been employed as an outcome measure in one study to test a natural analgesic agent against painful CIPN in 20 
thalidomide-treated and bortezomib-treated multiple myeloma (MM) patients. Results showed that pain and neurophysiological measures studied in lower limbs significantly improved. ${ }^{590}$

The main limitations of the technique are the relatively scarce availability of laser stimulators in most institutions and the sensitivity of LEPs to cognitive factors, in that they are modulated by attention, both factors hampering their use for widespread diagnostic purposes. Thus, further studies of LEPs are needed before they could find a place in routine CIPN assessment.

\section{CORNEAL CONFOCAL MICROSCOPY}

Corneal confocal microscopy (CCM) provides information on small sensory fibres of the trigeminal nerve by direct observation of corneal innervation in Bowman's layer. This technique has been demonstrated to be reproducible and useful in the evaluation of neuropathy associated with a variety of conditions. ${ }^{4,991}$

However, knowledge of the effect of chemotherapy on corneal nerves of CIPN patients is restricted to a cross-sectional study of 26 patients, ${ }^{592}$ and 2 prospective case-control stud-

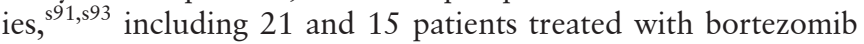
and oxaliplatin/cisplatin, respectively. Moreover, heterogeneous CIPN assessments were utilised in these studies ranging from the National Cancer Institute toxicity criteria ${ }^{\mathrm{s} 2}$ to more accurate diagnostic tests, including total neuropathy score, $\mathrm{NCS}^{591}$ and QST. ${ }^{593}$ In two of these studies, lower corneal nerve fibre density, nerve branch density and nerve fibre length was observed in cancer patients compared with control subjects, regardless of the presence of neuropathy, ${ }^{592,93}$ even at baseline, highlighting the high prevalence of pre-existing and/or subclinical CCM abnormalities in cancer. A reduction of the number of fibres and their length and density after chemotherapy, without alterations in the number of beadings or tortuosity, together with lack of correlation between CCM and clinical/neurophysiology outcomes, were reported. ${ }^{\mathrm{s} 91}$ Additional studies and wider availability are needed before CCM can be used as a tool to diagnose small fibre involvement in CIPN.

\section{NERVE IMAGING TECHNIQUES}

Nerve high-resolution ultrasound (HRUS) and magnetic resonance neurography (MRN) usually complement clinical and NCS in peripheral neuropathies. ${ }^{4,994}$ The main nerve HRUS measure is cross-sectional area (CSA)., ${ }^{4,94}$ CSA is generally increased in demyelinating neuropathy and normal or decreased in axonal pathology ${ }^{, 95}$ but this is not always straightforward. ${ }^{596}$ MRN evaluates nerve size, morphology, fascicular structure and perineurial-endoneurial fluid. Abnormal nerves may appear hyperintense and enlarged because of increased water. ${ }^{4}$ Diffusion tensor imaging (DTI) may offer quantitative information on nerve microstructure through fractional anisotropy (FA), diffusivity and apparent diffusion coefficient (ADC). ${ }^{.97}$

A small cross-sectional HRUS study showed increased CSA at common entrapment sites with no NCS evidence of entrapment in 9/15 oxaliplatin-treated patients, of whom 13 developed sensory axonal neuropathy. ${ }^{598}$ A prospective HRUS study on 13 oxaliplatin-treated patients confirmed increased CSA at upperlimb entrapment sites and documented tibial and fibular nerve CSA enlargement, which developed parallel or prior to clinical and NCS changes with no correlation between HRUS and NCS abnormalities. ${ }^{99}$ A pilot DTI study on the tibial nerve at the ankle in nine cancer patients found no FA or ADC difference between subjects with and without CIPN, but some correlations with vibratory perception threshold..$^{\text {s100 }}$
MRN may be helpful in suspected CIPN cases, and may show normal or mildly enlarged roots, plexuses and nerves, with no contrast enhancement. ${ }^{\text {s101 }} \mathrm{A}$ spinal MRI study showed lumbar root enhancement in 2/5 bortezomib-treated patients with $\mathrm{MM}$, who developed a peripheral neuropathy with severe motor involvement. ${ }^{\text {s102 }}$ An MRN-DTI study showed significant DRG hypertrophy, minor morphological qualitative alterations of peripheral nerves, non-significant diffusivity decrease and no FA changes in 20 patients with oxaliplatin-induced peripheral neuropathy. ${ }^{\text {s103 }}$

Central nervous system MRI may also further the understanding of CIPN pathogenesis. Spinal cord MRI showed posterior columns abnormality without cord swelling or contrast enhancement in a single patient with thalidomide-induced neuropathy, suggesting DRG neuronopathy, ${ }^{\mathrm{s} 104}$ but this finding was later confirmed only in $1 / 6$ patients. ${ }^{\text {sios }}$ A functional MRI study in $12 \mathrm{MM}$ patients and 12 healthy controls documented a characteristic activation pattern associated with painful $\mathrm{CIPN}^{\mathrm{s} 106}$; however, the view that MRI can offer, specific 'pain signatures', is debated. ${ }^{\text {s106, } 107}$ Moreover, performing functional MRI instead of asking patients to rate pain with clinical scales would be unpractical and very expensive in the clinical setting.

Being non-invasive, HRUS may be administered in clinical practice to document nerve changes during CIPN development, and to offer surrogate markers of nerve damage in future neuroprotective trials, overcoming the compliance issues related to repeated NCS testing. ${ }^{59}$ MRN and DTI might explore more proximal nerve sites and the spinal cord that HRUS cannot access, but such techniques are expensive and require a long post-processing time. ${ }^{.100}$ Table 2 describes the studies evaluating nerve imaging techniques in CIPN.

\section{CONCLUSIONS AND RECOMMENDATIONS}

A range of neurophysiological and other quantitative techniques (table 3 ) are now available to assess CIPN from its earlier stages, to provide insights into its mechanisms and for prospective or neuroprotection studies. However, in clinical practice, the vast majority of CIPN monitoring and management is performed using clinical examinations and patient-reported or clinicianreported outcomes. Patient-reported outcomes (PROs) form an essential adjunct to objective neurological assessmentsproviding information on the significance of symptoms and the patient experience. There are a number of validated PROs specific to CIPN, ${ }^{3}$ which have been widely translated to the clinical trial setting in recent years, although less broadly utilised in routine clinical practice. Further, recent recommendations for the structure of clinical trials in CIPN have provided a framework for future studies, including the important role of PROs as outcome measures. ${ }^{\mathrm{s} 108, \mathrm{~s} 109}$ However, there have not been specific recommendations made on the role of objective neurophysiological assessment in CIPN.

While NCS remain the key tool for the diagnosis of peripheral neuropathy, in the clinical oncology setting, the use of such techniques is often limited by the need for specialised training and equipment. However, NCS aids in diagnosis at subclinical and early symptomatic stages, assesses the extent and severity of large nerve fibre damage, and may also be used to monitor long-term outcomes. Most of the widely used neurotoxic chemotherapy agents damage large myelinated nerve fibres and hence standard NCS (incorporating assessment of sural or DSN amplitude) can provide useful prognostic information. While PRO measures are more commonly used as primary endpoints in CIPN neuroprotection trials, it is also imperative that neurologic 
examination and objective neurophysiological techniques, such as NCS, should be utilised in such clinical trials to determine neuroprotective efficacy and identify the mechanistic basis of symptomatic improvement.

Similarly, QSNEs provide useful information via easily applied methods that correlate well with NCS abnormalities. As such, their use in combination with NCS would be recommended as the best available evidence-based quantitative techniques for CIPN assessment. Incorporating a simple and easily applied test of sudomotor function in the lower limbs, such as SSR, may provide further valuable information on the possible involvement of autonomic or sympathetic neurons, besides that of somatic neurons, in CIPN.

Other neurophysiological specialised techniques have shed additional light into the pathophysiologic mechanisms of CIPN, and may provide further in-depth objective evidence of fibre damage for CIPN prediction, early detection and follow-up studies. Specifically, QST provides complementary and, in some cases, prognostic information regarding CIPN onset and progression. Nerve excitability techniques have provided information regarding the early development of CIPN, particularly in oxaliplatin-treated patients. Nerve imaging techniques with MRI and HRUS can be used to provide information on an increased CSA or increased nerve intensity in MRN, but this is not directly related to large fibre damage. Indeed, it is unclear whether CSA increase depends on the damage to $\mathrm{A} \beta, \mathrm{A} \delta$ or $\mathrm{C}$ fibres, or to the connective structures or vessels of the nerve. Moreover, nerve imaging techniques are useful in showing dorsal column pathological changes. Skin biopsy, CCM, LEPs, contact heat-evoked potentials and microneurography can also be applied to reveal the damage to small unmyelinated nerve fibres. Nevertheless, for some of the techniques reported above, the evidence of usefulness for the diagnosis of CIPN comes from small samples and/or single case reports, and thus should be considered as very preliminary and/or experimental. Accordingly, these techniques would have to provide reliable information as prognostic markers to be recommended in routine practice for patients under chemotherapy, especially since some of them cause discomfort, are time-consuming and increase the cost of patient management.

In any case, the further elaboration of such specialised techniques or refinement of classical neurophysiological techniques may identify objective neurophysiological biomarkers, whose inclusion might be helpful in neuroprotection clinical trials to monitor and quantify CIPN, to facilitate comparison between clinical studies and to strengthen the validity of the results based on PROs. ${ }^{\text {s110 }}$ Nevertheless, the predictive usefulness of conventional NCS requires independent validation sets and novel neurophysiological techniques need further study with robust design and sample sizes. Moreover, studies exploring the combination of NCS plus additional techniques are limited.

Taken in total, the use of multimodal testing is recommended as the optimal CIPN assessment strategy, employing objective NCS and other specialised techniques together with subjective PRO measures to reveal the full extent of CIPN abnormalities as well as to provide precise quantification of neuroprotection in clinical trials.

Additional 74 references of this document can be seen in the supplemental online-only data.

\footnotetext{
Author affiliations

${ }^{1}$ Neurology, "Saint Andrew's" State General Hospital of Patras, Patras, Greece

${ }^{2}$ The University of Sydney, Brain and Mind Centre, Faculty of Medicine and Health, Sydney, New South Wales, Australia

3icddr, b, International Centre for Diarrhoeal Disease Research, Bangladesh (icddr, b), Bangladesh
}

${ }^{4}$ Department of Neurosciences, Biomedicine and Movement Sciences, University of Verona, Verona, Italy

${ }^{5}$ Unit of Neuro-Oncology, Hospital Universitari de Bellvitge-ICO L'Hospitalet-IDIBELL, Barcelona, Spain

${ }^{6}$ Experimental Neurology Unit and Milan Center for Neuroscience, University of Milano-Bicocca, Monza, Italy

${ }^{7}$ Unit of Neuro-Oncology, Hospital Universitari de Bellvitge-ICO L'Hospitalet-IDIBELL, Barcelona, Spain

${ }^{8}$ Service de Neurologie 2-Mazarin, Hôpitaux Universitaires La Pitié Salpêtrière Charles Foix, APHP, Paris, France

${ }^{9}$ Experimental Neurology Unit and Milan Center for Neuroscience, University of Milan Bicocca, Monza, Italy

${ }^{10}$ Neurology, Johns Hopkins University School of Medicine, Baltimore, Maryland, USA

Acknowledgements This review paper was produced by the members of the Clinical Working Group of the Toxic Neuropathy Consortium (TNC), representing the core TNC of the Peripheral Nerve Society. However, the members of the core TNC not quoted in the authors' list and are not qualified to be named as authors, contributors, collaborators or co-investigators of this manuscript.

Contributors AAA and SBP: performed the literature search, selected the relevant articles, wrote the paper and led the discussion. BI, ST, PA, JB, RV and DP: performed the literature search, selected the relevant articles and wrote the paper. GC and DRC: wrote the paper, led the discussion and edited the paper.

Funding This research has received no specific grant from any funding agency in the public, commercial or not-for-profit sectors.

Competing interests GC serves as a consultant to PledPharma AB, Helsinn, Disarm, Rottapharm and Toray. He is a non-voting member of the board of directors of the Peripheral Nerve Society and chair of the Toxic Neuropathy Consortium. DRC is a consultant to Annexon Biosciences, argenx SE, Biotest Pharmaceuticals, Inc., Cigna Health Management, Inc., CSL Behring, DP Clinical, Inc., Grifols S.A., Hansa Medical AB, New Enterprise Associates, Inc., Octapharma AG, Pharnext SAS, Polyneuron Pharmaceuticals, Seattle Genetics Inc., Stealth BioTherapeutics and Syntimmune, Inc.; is a member of the Data Safety Monitoring Board for Pfizer, Inc., Sanofi S.A., Alnylam Pharmaceuticals, PledPharma AB and Momenta Pharma; has technology licensing agreements through Johns Hopkins University with AstraZeneca Pharmaceuticals, LP, Genentech, Inc., Levicept, Inc., Seattle Genetics, Inc., Merrimack Pharmaceuticals, Levicept Limited and Disarm Therapeutics, Inc.; is a non-voting member of the board of directors of the Peripheral Nerve Society and is editor-in-chief at Journal of the Peripheral Nervous System (until 26 June 2019). AAA, SBP, BI, ST, RV, PA, JB and DP do not have any conflicts of interest or financial disclosures to declare in relation to this manuscript.

Patient consent for publication Not required.

Provenance and peer review Not commissioned; externally peer reviewed.

\section{ORCID iDs}

Andreas A Argyriou http://orcid.org/0000-0003-2131-7114

Stefano Tamburin http://orcid.org/0000-0002-1561-2187

\section{REFERENCES}

1. Shah A, Hoffman EM, Mauermann ML, et al. Incidence and disease burden of chemotherapy-induced peripheral neuropathy in a population-based cohort. J Neurol Neurosurg Psychiatry 2018;89:636-41.

2. Park SB. Chemotherapy-induced peripheral neuropathy: highlighting unmet needs. J Neurol Neurosurg Psychiatry 2018;89.

3. Kandula T, Farrar MA, Kiernan MC, et al. Neurophysiological and clinical outcomes in chemotherapy-induced neuropathy in cancer. Clin Neurophysiol 2017;128:1166-75.

4. Gasparotti R, Padua L, Briani C, et al. New Technologies for the assessment of neuropathies. Nat Rev Neurol 2017;13:203-16.

5. McDonald ES, Windebank AJ. Cisplatin-induced apoptosis of DRG neurons involves Bax redistribution and cytochrome cRelease but not Fas receptor signaling. Neurobiol Dis 2002;9:220-33.

6. Dasari S, Tchounwou PB. Cisplatin in cancer therapy: molecular mechanisms of action. Eur J Pharmacol 2014;740:364-78.

7. Webster RG, Brain KL, Wilson RH, et al. Oxaliplatin induces hyperexcitability at motor and autonomic neuromuscular junctions through effects on voltage-gated sodium channels. Br J Pharmacol 2005; 146:1027-39.

8. Park SB, Goldstein D, Lin CS-Y, et al. Acute abnormalities of sensory nerve function associated with oxaliplatin-induced neurotoxicity. J Clin Oncol 2009;27:1243-9.

9. Jordan MA, Wilson L. Microtubules as a target for anticancer drugs. Nat Rev Cancer 2004; 4:253-65.

10. Lobert S, Vulevic B, Correia JJ. Interaction of vinca alkaloids with tubulin: a comparison of vinblastine, vincristine, and vinorelbine. Biochemistry 1996;35:6806-14.

11. Landowski TH, Megli CJ, Nullmeyer KD, et al. Mitochondrial-mediated disregulation of $\mathrm{Ca} 2+$ is a critical determinant of Velcade (PS-341/bortezomib) cytotoxicity in myeloma cell lines. Cancer Res 2005;65:3828-36. 
12. Kiernan MC, Bostock H. Effects of membrane polarization and ischaemia on the excitability properties of human motor axons. Brain 2000;123:2542-51.

13. Argyriou AA, Cavaletti G, Bruna J, et al. Bortezomib-induced peripheral neurotoxicity: an update. Arch Toxicol 2014;88:1669-79.

14. Zara G, Ermani M, Rondinone R, et al. Thalidomide and sensory neurotoxicity: a neurophysiological study. J Neurol Neurosurg Psychiatry 2008;79:1258-61.

15. Flatters SJL, Dougherty PM, Colvin LA. Clinical and preclinical perspectives on chemotherapy-induced peripheral neuropathy (CIPN): a narrative review. Br J Anaesth 2017;119:737-49.

16. Fuglsang-Frederiksen A, Pugdahl K. Current status on electrodiagnostic standards and guidelines in neuromuscular disorders. Clin Neurophysiol 2011;122:440-55.

17. Krarup-Hansen A, Helweg-Larsen S, Schmalbruch H, et al. Neuronal involvement in cisplatin neuropathy: prospective clinical and neurophysiological studies. Brain 2007;130:1076-88.

18. Argyriou AA, Polychronopoulos P, Iconomou G, et al. Incidence and characteristics of peripheral neuropathy during oxaliplatin-based chemotherapy for metastatic colon cancer. Acta Oncol 2007;46:1131-7.

19. Briani C, Argyriou AA, Izquierdo C, et al. Long-term course of oxaliplatin-induced polyneuropathy: a prospective 2-year follow-up study. J Peripher Nerv Syst 2014;19:299-306.

20. Argyriou AA, Polychronopoulos P, Koutras A, et al. Clinical and electrophysiological features of peripheral neuropathy induced by administration of cisplatin plus paclitaxel-based chemotherapy. Eur J Cancer Care 2007;16:231-7.

21. Chen X, Stubblefield MD, Custodio CM, et al. Electrophysiological features of taxane-induced polyneuropathy in patients with breast cancer. J Clin Neurophysiol 2013;30:199-203.

22. Velasco R, Petit J, Clapés V, et al. Neurological monitoring reduces the incidence of bortezomib-induced peripheral neuropathy in multiple myeloma patients. J Peripher Nerv Syst 2010;15:17-25.

23. Kocer B, Sucak G, Kuruoglu R, et al. Clinical and electrophysiological evaluation of patients with thalidomide-induced neuropathy. Acta Neurol Belg 2009;109:120-6.

24. Lavoie Smith EM, Li L, Chiang C, et al. Patterns and severity of vincristine-induced peripheral neuropathy in children with acute lymphoblastic leukemia. J Peripher Nerv Syst 2015;20:37-46

25. Mileshkin L, Stark R, Day B, et al. Development of neuropathy in patients with myeloma treated with thalidomide: patterns of occurrence and the role of electrophysiologic monitoring. J Clin Oncol 2006;24:4507-14.
26. Thawani SP, Tanji K, De Sousa EA, et al. Bortezomib-associated demyelinating neuropathy--clinical and pathologic features. J Clin Neuromuscul Dis 2015;16:202-9.

27. Argyriou AA, Koltzenburg M, Polychronopoulos $P$, et al. Peripheral nerve damage associated with administration of taxanes in patients with cancer. Crit Rev Oncol Hematol 2008;66:218-28.

28. Argyriou AA, Polychronopoulos P, Koutras A, et al. Peripheral neuropathy induced by administration of cisplatin- and paclitaxel-based chemotherapy. could it be predicted? Support Care Cancer 2005;13:647-51.

29. Velasco R, Bruna J, Briani C, et al. Early predictors of oxaliplatin-induced cumulative neuropathy in colorectal cancer patients. J Neurol Neurosurg Psychiatry 2014;85:392-8.

30. Cavaletti G, Tredici G, Petruccioli MG, et al. Effects of different schedules of oxaliplatin treatment on the peripheral nervous system of the rat. Eur J Cancer 2001;37:2457-63.

31. Dalla Torre C, Zambello R, Cacciavillani M, et al. Lenalidomide long-term neurotoxicity: clinical and neurophysiologic prospective study. Neurology 2016;87:1161-6.

32. Koçer A, Domaç FM, Boylu E, et al. A comparison of sural nerve conduction studies in patients with impaired oral glucose tolerance test. Acta Neurol Scand 2007;116:399-405.

33. Turgut $B$, Turgut $N$, Akpinar $S$, et al. Dorsal sural nerve conduction study in vitamin $B$ (12) deficiency with megaloblastic anemia. J Peripher Nerv Syst 2006;11:247-52.

34. Uluc K, Isak B, Borucu D, et al. Medial plantar and dorsal sural nerve conduction studies increase the sensitivity in the detection of neuropathy in diabetic patients. Clin Neurophysiol 2008;119:880-5.

35. Alberti P, Rossi E, Argyriou AA, et al. Risk stratification of oxaliplatin induced peripheral neurotoxicity applying electrophysiological testing of dorsal sural nerve. Support Care Cancer 2018;26:3143-51.

36. Wehrwein EA, Orer HS, Barman SM. Overview of the anatomy, physiology, and pharmacology of the autonomic nervous system. Compr Physiol 2016;6:1239-78.

37. Adams SC, Schondorf R, Benoit J, et al. Impact of cancer and chemotherapy on autonomic nervous system function and cardiovascular reactivity in young adults with cancer: a case-controlled feasibility study. BMC Cancer 2015;15.

38. Argyriou AA, Koutras A, Polychronopoulos $P$, et al. The impact of paclitaxel or cisplatinbased chemotherapy on sympathetic skin response: a prospective study. Eur I Neurol 2005;12:858-61.

39. Saad M, Psimaras D, Tafani C, et al. Quick, non-invasive and quantitative assessment of small fiber neuropathy in patients receiving chemotherapy. J Neurooncol 2016;127:373-80. 